\title{
AVANCES DISCIPLINARIOS EN LAS RELACIONES INTERNACIONALES: LA DEFINICIÓN DE ACTOR INTERNACIONAL EN EL ESTUDIO DE LA PARADIPLOMACIA
}

\author{
DISCIPLINARY PROGRESS IN INTERNATIONAL RELATIONS: \\ THE DEFINITION OF INTERNATIONAL \\ ACTOR IN THE STUDY OF PARADIPLOMACY
}

\author{
Nahuel Oddone ${ }^{1}$ \\ ORCID: 0000-0002-3120-3914 \\ Mariana Luna Pont ${ }^{2}$ \\ ORCID: 0000-0002-8806-6471
}

\section{RESUMEN:}

El aniversario del nacimiento como disciplina encuentra al estudio de las Relaciones Internacionales en una etapa de revisión y reinterpretación de su trayectoria. La revisión en curso aborda la epistemología, teorías, debates y agendas de investigación, al tiempo que se coloca el foco en la influencia de contextos y actores en el desarrollo de la disciplina, entre ellos de los gobiernos locales y regionales. En este trabajo se estudian textos y contextos referidos a la forma en que la teoría de las relaciones internacionales ha investigado el desempeño internacional subnacional. En particular, se analiza la forma en que tales desempeños han ingresado al campo de estudio desde su etapa fundacional, para concentrar la atención en la revisión de la categoría actor internacional en el estudio de la paradiplomacia.

Palabras clave: Relaciones Internacionales; actor internacional; paradiplomacia; gobiernos locales.

\section{ABSTRACT:}

The commemoration of the foundation of International Relations as a scientific discipline, finds the analysis of International Relations in a stage of review and reinterpretation of its trajectory. The ongoing assessment addresses issues such as epistemology, theories, debates, and research agendas focusing on the influence of contexts and stakeholders in the development of the discipline, including local and regional governments. This article alludes to texts and contexts appraising the way in which the theory of International Relations has analyzed subnational international performance. In particular, the way in which such activities have entered this field of study since its founding stage, paying attention to the revision of the category of international actor in the study of paradiplomacy.

Keywords: International Relations; International Actor; Paradiplomacy; Local Goverments.

1 Instituto Social del Mercado Común del Sur, Departamento de Promoción e Intercambio de Políticas Sociales, Paraguay. Jefe del Departamento y Doctor en Estudios Internacionales. Las opiniones aquí reflejadas son de estricto carácter personal y pueden no coincidir con su institución de pertenencia. Correo electrónico: oddone.nahuel@gmail.com

2 Universidad Nacional Tres de Febrero, Departamento de Maestría en Integración Latinoamericana, Argentina. Licenciada en Relaciones Internacionales. Coordinadora Académica y Licenciada en Relaciones Internacionales. Correo electrónico: mulunapont@gmail.com 


\section{Introducción}

El aniversario del nacimiento como disciplina encuentra al estudio de las relaciones internacionales en una etapa de revisión y reinterpretación de su trayectoria. La revisión en curso aborda la epistemología, teorías, debates y agendas de investigación, al tiempo que se coloca el foco en la influencia de contextos y actores en el desarrollo de la disciplina, entre ellos de los gobiernos locales y regionales.

En la literatura especializada se suele asumir que, si bien la proyección internacional subnacional -la denominada paradiplomacia-, no es fenómeno novedoso, ingresa tardíamente al campo de estudio de las relaciones internacionales. Una lectura más atenta, fundada en desarrollos recientes, permite reparar en que tal proyección internacional cuenta con una extensa tradición e influencia, ya que desde inicios del siglo XX se asiste a la conformación de las primeras asociaciones internacionales de ciudades y municipios; así como a la institucionalización de conferencias y mecanismos de cooperación intermunicipal -en los que la reflexión y movilización en torno de lo internacional está presente- que coinciden, en el tiempo, con el surgimiento de las Relaciones Internacionales como campo de estudio (Luna Pont, 2019).

Por otra parte, los estudios sobre paradiplomacia, durante largo tiempo, han concentrado su atención en el análisis de casos por países y, particularmente, en el marco de Estados federales. Mucho se ha discutido acerca de si tales proyecciones constituyen políticas exteriores o manifestaciones de políticas internas que se internacionalizan o, más recientemente, en particular a partir de la década de 1990, como un tipo de respuesta a los desafíos del proceso de globalización en curso y a los dilemas de la gobernanza global.

En este trabajo se abordan textos y contextos en los que la paradiplomacia ingresa al campo de estudio de las relaciones internacionales y la forma en que la teoría de las Relaciones Internacionales da cuenta de ello. En particular, se concentra la atención en las perspectivas que abordan la paradiplomacia desde la categoría actor internacional. Para ello la exposición está organizada en tres partes.

En primer lugar -valiéndose de la introducción de perspectivas críticas en la historia de la disciplina y de la difusión de enfoques de historia intelectual que proponen una revisión del contexto, ideas, referentes e instituciones que han operado en la conformación del campo y el itinerario recorrido-se investigan dinámicas de proyección internacional subnacional en el contexto de la conformación de las relaciones internacionales como campo de estudio en 
tanto voces y prácticas que, por largo tiempo, han quedado en los "márgenes" de la disciplina.

En una segunda parte se aborda el debate propio de la paradiplomacia en la entrada del siglo XX y su impacto en la disciplina de las relaciones internacionales en cuanto corpus teórico que ha estudiado especialmente el accionar internacional de los Estados centrales de base soberana. Finalmente, en la tercera sección, se analiza la forma en que la teoría de las relaciones internacionales da cuenta del fenómeno de la paradiplomacia desde la perspectiva del "actor internacional". Definir y comprender dicha categoría -quién es considerado actor internacional, o quién reúne las cualidades necesarias para ser considerado como tal- ha sido una constante preocupación para la evolución teórica de la disciplina y la ampliación de su capacidad de dar cuenta de la complejidad de la política mundial (García Segura, 1993), y la paradiplomacia introduce, en este sentido, nuevos desafíos para la comprensión y manejo de los asuntos globales.

\section{Un rescate de las narrativas sobre la paradiplomacia en el siglo XIX}

La identidad de las Relaciones Internacionales como disciplina es portadora de una autoimagen y un conjunto de mitos fundacionales que marcaron su trayectoria y se han mantenido relativamente incuestionados por largo tiempo. La primera imagen o interpretación remite, esencialmente, al contexto temporal y geográfico del surgimiento como campo de estudio, al lugar central otorgado a las relaciones interestatales y a la evolución a partir de una sucesión de grandes debates teóricos.

Ha sido ampliamente aceptado que tiene su génesis como reacción a los horrores de la Primera Guerra Mundial y a la necesidad de prevenir futuras conflagraciones. Una segunda interpretación identifica como primer espacio académico de estudio a la cátedra de Política Internacional -con la dirección de Alfred Zimmern- creada en la Universidad de Gales (hoy Aberystwyth) en el año $1919^{3}$, y el debate entre aproximaciones idealistas y realistas durante el período de entreguerras. Este debate constituyó el primero de una serie de debates interparadigmáticos que marcarían la trayectoria y evolución de la disciplina.

La conmemoración del centenario de su nacimiento encuentra al estudio de las relaciones internacionales en una etapa de revisión y reinterpretación de las imágenes y de aquellos mitos fundacionales. La introducción de perspectivas críticas en la historia de la disciplina y la difusión de enfoques de historia intelectual

3 En 1920 se creó en Londres el Royal Institute of International Affairs y al año siguiente en Nueva York el Council of Foreign Relations. 
proponen una revisión del contexto, ideas, referentes e instituciones que han operado en la conformación del campo y el itinerario recorrido. Podría afirmarse que entre las revisiones en curso se encuentran dos abordajes predominantes. Por un lado, aquel centrado en la influencia de factores ideacionales: epistemología, teorías, enfoques, ideas, debates y agendas de investigación; y, por el otro, el de quienes colocan el foco en la influencia de contextos y actores en el desarrollo de la disciplina, vale decir, en las denominadas fuerzas materiales que incluyen a los gobiernos locales y regionales. En este texto se hace hincapié en la categoría de actor en el estudio de la internacionalización de los gobiernos subnacionales o subestatales, comúnmente conocida como paradiplomacia.

Ejemplo del primer tipo de aproximación es el cuestionamiento de la pretensión universal, en el espacio y en el tiempo, de los marcos conceptuales de referencia y enfoques teóricos dominantes, fundamentalmente aquellos de origen occidental. Tal como sostiene Rubiolo (2019), la demanda de pluralismo y diversidad enarbolada por Acharya y Buzan (2010 y 2019)-ha adquirido centralidad y pone en cuestión la capacidad explicativa de los enfoques tradicionales. Manifiesta la necesidad de apartarse de la arraigada perspectiva anglosajona dominante, para dar lugar a una revalorización de las trayectorias locales y al reconocimiento de las diferentes expresiones de los fenómenos globales en distintas latitudes, que dan lugar a historias, narrativas y conceptualizaciones diversas, no reconocidas por el mainstream que ha dominado los saberes (Rubiolo, 2019).

Estos autores invitan a indagar sobre aquellas voces, problemáticas y perspectivas que han quedado en los "márgenes" de la disciplina, en las ausencias y exclusiones temáticas. Tussie (2019) ha puesto de relieve que la ampliación de la deliberación cognitiva en el debate de las relaciones internacionales ha recorrido un largo camino con una creciente incorporación de nuevos temas, en donde la problematización del desarrollo ganó espacio a partir de la resonancia de algunas voces periféricas. Estas modificaciones epistemológicas impulsaron la investigación y enseñanza prospectiva (Batta Fonseca, 2013). Los alcances propios de esta revisión aún están abiertos.

Reflejo de la segunda perspectiva es la reinterpretación de las circunstancias propias del surgimiento de la disciplina, rastreándolo hasta el último tramo del siglo XIX, contexto asociado a las consecuencias de complejos procesos económicos, sociales, políticos y culturales que venían operando desde la Revolución Industrial, y a la reacción a la expansión económica -asociada a cambios científicos y tecnológicos, la revolución en los medios de comunicación y la mayor interconexión global-y a la competencia interestatal que caracterizó el fin de siglo como el nacionalismo, la expansión imperialista, el creciente militarismo 
y belicismo, los cambios en el balance de poder y los nuevos poderes emergentes, entre otros factores.

La identificación de una "generación perdida de internacionalistas" que abordó las relaciones internacionales en las décadas precedentes a la Gran Guerra (Knutsen, 2008), cuyos aportes no fueron reconocidos por los paradigmas dominantes hasta muy recientemente, no solo afecta el marco temporal de surgimiento del campo de estudio, sino también al conjunto de temáticas y problemáticas que quedaron, en muchos casos, silenciadas en los desarrollos posteriores. Tales son los siguientes ejemplos: la administración colonial, la cooperación y el conflicto con las colonias y las relaciones de dependencia; la dimensión local de la expansión imperial y las empresas imperialistas de ciudades (hoy abordados como expresión de imperialismo municipal), la emergencia de la cooperación intermunicipal; la proliferación de asociaciones internacionales de carácter municipal y las otrora denominadas uniones internacionales y su articulación a través de redes, ferias y exposiciones universales; la incidencia de concepciones raciales en las relaciones internacionales; la relación entre nacionalismo, imperio y raza; la influencia del concepto de civilización; el papel de la ciencia y de las comunidades de expertos en la cooperación internacional y la búsqueda de la paz; entre otros temas.

No es casual que en esos años se desarrollara un cuerpo significativo de literatura que reclamaba conformar una "ciencia del internacionalismo" (Fied, 1909), en muchos casos reaccionando a la proliferación de asociaciones y uniones internacionales cuya proyección internacional era percibida como un indicador más de un mundo crecientemente interconectado e interdependiente:

La vida internacional conduce cada vez más la vida de las naciones. Todos los dominios manifiestan una constante interpenetración de ideas, necesidades e intereses, e interminables actividades intelectuales y materiales trascienden las fronteras. El siglo XX se anuncia como aquel que debe proporcionar a la comunidad civilizada una nueva estructura general, mejor adaptada a nuevas condiciones (...) A su vez, esta estructura tendrá en cuenta las relaciones entre las naciones y entre los intereses cada vez más especializados. Multiplicará las fuerzas de las colectividades y hará que el internacionalismo sea un factor de civilización. (Speeckaert, 1970, p. 22)

Progresivamente, a ambos lados del Atlántico, fue emergiendo una nueva representación de la cuestión social. La ciudad aparecía como causa de problemas sociales y también como origen de soluciones a través de su reorganización, para lo que se requirieron nuevos métodos de intervención y un papel más activo de los gobiernos locales. Las ciudades, las autoridades municipales y sus 
trayectorias de gobierno comenzaron a ser objeto de estudios comparativos, sobre la base de la observación e intercambios de conocimiento y de las experiencias de gestión. Las ferias, exposiciones y congresos internacionales fueron escenarios privilegiados para el intercambio y transferencia de ideas, prácticas e innovaciones donde los problemas locales y nacionales adquirían una dimensión internacional. Así, la cuestión urbana se fue internacionalizando.

Producto de esta dinámica, se asistió a la conformación de una comunidad epistémica de reformadores sociales cuyos miembros estaban interconectados a través de viajes, comunicaciones y la traducción y circulación de publicaciones. Este flujo de ideas y prácticas facilitó la emergencia de un discurso transnacional sobre las pautas de modernización urbana a escala europea y trasatlántica, y la conformación de redes de intercambio y asociaciones a un ritmo desconocido hasta entonces. Fue forjándose, así, un denso "movimiento municipalista" con conexiones internacionales -inicialmente europeas, luego noratlánticas y más tarde globales- constituido por una constelación de equipos políticos, técnicos, funcionarios, administradores, planificadores, reformistas urbanos y activistas políticos de diversas nacionalidades, culturas y afiliaciones políticas (Luna Pont, 2019).

Tal como se afirmaba en el Primer Congreso Internacional y Exposición de Ciudades realizado en Ghent, Bélgica, en el año 1913:

El progreso general de la civilización, el avance logrado por las naciones más atrasadas, significan que las mismas cuestiones son enfrentadas hoy por las autoridades gobernantes de todo el mundo. Las soluciones halladas por algunas de ellas pueden ser usadas por las demás, si no exactamente de la misma forma, al menos después de algunas adaptaciones necesarias. En consecuencia, es cada vez más probable que todos los interesados en el mejoramiento de los pueblos y sus servicios municipales, de la vida municipal, se beneficien del agrupamiento de su experiencia y del análisis de las posibles soluciones a la luz de amplias comparaciones. (Premier Congrés International et Exposition Compareé des Villes, 1914: p.viii)

Este intercambio generó una cantidad creciente de profesionales e instituciones que aspiraban a organizarlo y en ocasiones monopolizarlo. Se destacán tres organizaciones de alcance mundial: la Union Internationale des Villes (UIV) conocida como International Union of Local Authorities (IULA) desde 1928, creada en el año 1913 y orientada hacia la cooperación intermunicipal y el conocimiento científico de la administración local; la International Garden Cities and Town Planning Association, fundada ese mismo año, cuya principal línea de trabajo fue la planificación espacial urbana; y el International Institute of 
Administrative Sciences (IIAS), instaurado en 1910, focalizado en la difusión del conocimiento sobre las teorías, los métodos y las técnicas administrativas (Luna Pont, 2019).

Uno de los rasgos de este movimiento municipalista internacional fue, justamente, su orientación internacionalista. En su interior convivían diversas tendencias, si bien se destacaban dos grandes corrientes: la primera era portadora de un discurso esencialmente político que vinculaba el ideal de cooperación universal con el propósito político de transformación social y buscaba modelos ideales de aplicación universal como soluciones alternativas a los problemas de la sociedad contemporánea; y la segunda, más pragmática y técnica, enfocaba los vínculos intermunicipales y su proyección internacional, esencialmente como alternativas para el intercambio de información profesional, técnica y científica (Couperus, 2011, Luna Pont, 2019).

De esta manera, el gobierno local era percibido cada vez más como una institución crucial para la promoción no solo de modernización y progreso social, sino también como vehículo de cooperación internacional y de paz. En algunos casos se apelaba a la imagen de un mundo en el cual las ciudades serían "las celdas básicas de un orden democrático más dispuesto a la paz, el conocimiento mutuo y la resolución de los problemas sociales" más allá de las fronteras nacionales que separaban a los diferentes países (Fried, 1909, p. 36).

Una mirada atenta a estos desarrollos propone no solo una nutrida "agenda de reflexión" sobre temas internacionales desde el último cuarto del siglo XIX, sino también sobre la incidencia del desempeño internacional de actores no estatales y subnacionales desde la etapa fundacional de la disciplina, que ha permanecido oculta bajo la hegemonía de una concepción profundamente estatocéntrica de los estudios internacionales. Con el correr del siglo XX, la proyección internacional de ciudades y municipios fue adquiriendo otro carácter. $\mathrm{Su}$ mayor visibilidad se observa, sobre todo, desde la década del noventa del siglo pasado. Sin embargo, vale la pena reconocer que las principales asociaciones internacionales, lideradas por IULA, encontraron, al inicio, en la Sociedad de Naciones y, luego, en el sistema de las Naciones Unidas, escenarios relativamente complementarios para su desempeño; al tiempo que los acuerdos de hermanamiento de ciudades, la inserción en espacios de integración regional, la cooperación descentralizada y el trabajo en red crearon condiciones para que tales prácticas comenzaran a ser concebidas como políticas públicas.

Indudablemente, estas dinámicas de manera progresiva comenzaron a cambiar la fisonomía de las relaciones internacionales, al punto de comenzar a ser percibidas y estudiadas en el marco de la teoría de las relaciones internacionales 
como expresión de la emergencia de nuevos actores internacionales con capacidad de impacto en la dinámica de la política mundial y en el manejo de los asuntos globales, cuestiones que serán abordadas en las próximas secciones.

\section{Camino al multiparadigmatismo: La diversidad teoría de las relaciones internacionales y la paradiplomacia como objeto de estudio}

Lo que hoy se reconoce como Relaciones Internacionales ha recorrido un largo camino para su definición teórica.

Lo que hoy denominamos la disciplina de las relaciones internacionales ha recorrido un largo trayecto histórico, antes de su consideración como disciplina científica en el marco de las ciencias sociales. Tan largo que empieza con las primeras consideraciones e interpretaciones de la realidad internacional. Interpretaciones teóricas de una práctica internacional que se remonta en el tiempo más allá de la aparición del Estado soberano y de la constitución del sistema europeo de Estados. (Del Arenal, 1981, p. 852)

En las relaciones internacionales se han desarrollado muchos esfuerzos por sintetizar numerosas disciplinas tradicionales, como la historia o el derecho, cada una con un punto de vista especializado dentro de la "unidad" que constituye la sociedad internacional.

Lo que caracteriza las relaciones internacionales es, de un lado, su globalidad, en cuanto que pretende dar cuenta de la sociedad internacional en su totalidad y no sólo de alguno de los elementos y fenómenos que en ella se producen, y, de otro, aunque ello no siempre esté presente en todas las teorías de las relaciones internacionales, el énfasis que pone en el punto de vista propiamente internacional o, si se prefiere, de la sociedad internacional, frente al punto de vista que parte del Estado y de su proyección exterior como actor de las relaciones internacionales. (Del Arenal, 1981, p. 853)

De acuerdo con Holsti (2005), esta disciplina ha sido un campo de estudio donde "se combinan ideas, conceptos y metodologías procedentes de distintas fuentes. Por lo tanto, las verdades y las interpretaciones de las relaciones internacionales necesariamente difieren en función de las perspectivas teóricas y epistemológicas que se apliquen a determinado problema" (Holsti, 2005, p. 25). Fred Halliday (2002) sostiene que "los elementos constitutivos de las relaciones internacionales dan lugar a muchas especificidades y enfoques teóricos variados. Lo que provoca que la diversidad teoría de esta disciplina sea uno de sus puntos fuertes" (Halliday, 2002, p. 26). 
No es objeto de este artículo hacer un repaso de la historia de las escuelas que conforman los debates en los estudios de las relaciones internacionales. Sin embargo, es necesario poner de relieve que hasta mediados de los años sesenta existía un extendido consenso en sostener que el Estado era el único actor del sistema internacional. En palabras de Krippendorff (1993): "Hablar de 'política internacional' significa hablar de Estado. Parece una verdad sobreentendida que el Estado constituye el verdadero objeto de las relaciones internacionales, su 'actor' y 'autor de las acciones"' (Krippendorff, 1993, p. 65). Desde una perspectiva similar, Renouvin y Duroselle (2000) afirmaban:

El estudio de las relaciones internacionales se ocupa sobre todo de analizar y de explicar los tratos entre las comunidades políticas organizadas dentro de un territorio, es decir, entre los Estados. ... debe tenerse en consideración los vínculos establecidos entre los pueblos y entre los individuos ...: el intercambio de productos y de servicios, las comunicaciones de ideas, el juego de las influencias recíprocas ..., las manifestaciones de simpatía o antipatía. Pero reconoce que rara vez estas relaciones pueden disociarse de las que se han establecido entre los Estados ... . Reguladas por los Estados, se convierten en un elemento de negociación o de conflictos entre los gobiernos. Por tanto, es la acción de los Estados la que se encuentra en el centro de las relaciones internacionales (Renouvin y Duroselle, 2000, p. 9).

Las visiones estatocéntricas comenzaron a fragmentarse parcialmente, a partir de los años sesenta, cuando las unidades subnacionales quedan:

asumidas como actores relevantes de la política mundial y las relaciones internacionales generadas desde esa escala comienzan a recibir un tratamiento específico y pormenorizado, favorecido por la emergencia de una nueva retórica acerca de lo local y de perspectivas que revisan las concepciones tradicionales de espacialidad y territorialidad que habían limitado la perspectiva disciplinaria de las relaciones internacionales y de las ciencias sociales en general. (Luna Pont, 2010, p. 56)

Por ello, en los enfoques posteriores al transnacionalismo, esta justificación es de tipo funcional, a partir del proceso por el cual la entidad subnacional reúne la capacidad de movilizar recursos para alcanzar objetivos y ejercer influencia en el sistema internacional (Russell, 2010).

El grado de autonomía del actor subestatal frente al gobierno central será una de las características principales que será analizada por el transnacionalismo. Desde esta lógica funcional, la valorización del actor internacional se da a partir de la "habilidad de una entidad para provocar consecuencias en la política internacional sin que su comportamiento sea predecible a partir de la referencia 
a otros actores" (García Segura, 1993, p. 18). La autonomía de los gobiernos subnacionales "no implica una atomización del poder del Estado sino, por el contrario, una mayor potestad en la coordinación y gestión de los recursos, bajo una idea de poder compartido" (Oddone, 2008, p. 30).

Existe, por tanto, cierto consenso en la academia especializada en identificar la perspectiva de la definición del actor internacional como uno de los encuadres teóricos privilegiados para estudiar la paradiplomacia (García Segura, 1992 y 1993; Luna Pont, 2010 y 2011; Oddone, 2016 y 2019; Lara, 2019). Como ha sostenido Luna Pont, "el predominio de visiones estatocéntricas, fundamentalmente jurídicas y formales, centradas en el modelo westfaliano de vinculaciones externas de los Estados, ha contribuido a brindar un encuadre limitado de la naturaleza y orientaciones de la política mundial" (2010, p. 51). García Segura (1993) hace hincapié en el papel del transnacionalismo como un parteaguas en la interpretación del actor internacional. Esta etapa de enfoque russelliano se corresponde con la etapa del pluralismo en la disciplina, también conocida como "tercer debate" o "debate interparadigmático". Tal como sostiene Waever: "No sólo había más actores que el Estado, el Estado ya no era el Estado, sino que tenía que ser descompuesto en redes de burocracias, grupos de interés e individuos en una perspectiva pluralista" (1996, p. 350).

Tal como resalta Díaz Abraham (2005), es importante establecer algunas condiciones comunes a las distintas líneas de pensamiento dentro de las principales corrientes teóricas de las Relaciones Internacionales con respecto a la concepción de la naturaleza y la definición de objetivos dentro de su teoría a lo largo de sus distintas etapas, comúnmente conocidas como debates interparadigmáticos. La evolución de esta "condición ha derivado en que el debate inter-paradigmático ha dado paso al multiparadigmatismo, como una forma menos constreñida de dar sentido a la realidad internacional, ya que concilia de manera poco dogmática algunos elementos teóricos de las diferentes corrientes de pensamiento" (Díaz Abraham, 2005, p. 27), incluyendo en esta conciliación de perspectivas "la evolución del transnacionalismo hacia posiciones compatibles con el neorrealismo" (Sodupe, 2003, p. 52).

En las últimas décadas, se han replanteado los esquemas clásicos ante "la mayor complejidad de las relaciones internacionales ante la emergencia de nuevos y más plurales actores, la generalización de procesos democráticos y descentralizadores en el mundo, el renacimiento de las dinámicas políticas y sociales de base territorial, y la generación de nuevas visiones sobre el desarrollo" (Romero, 2004, p. 19). Esta complejidad se correspondió con modificaciones sustantivas dentro de la disciplina que perseguían el análisis y la comprensión de la nueva realidad. Estas modificaciones incluyeron el acercamiento con otras disciplinas, 
sobre todo, aquellas centradas en el análisis de las administraciones públicas locales, sus procesos de negociación interna y de toma de decisiones. Las disciplinas que estudiaban los "aspectos internos" de los gobiernos, la funcionalidad de sus burocracias, fueron pronto incluidas en el análisis internacional.

El enfoque transnacionalista desarrollado a inicios de la década de 1970 (Keohane y Nye, 1974, Kaiser, 1969; Rosenau, 1969; Young, 1971) introdujo un cuestionamiento a tres supuestos fundamentales de la escuela realista, que dominaba la teoría de las Relaciones Internacionales hasta entonces: en primer lugar, que los Estados centrales son los únicos actores del sistema de relaciones internacionales; en segundo lugar, que estos se proyectan sobre la esfera internacional de manera integrada y monolítica sin fisuras internas en la representación del interés nacional, y en tercer lugar, que su actuación internacional siempre se corresponde con un modo de actuar racional (Rolandi y Merello, 2016; Russell, 2010). Desde la perspectiva de Díaz Abraham "hay que señalar que el transnacionalismo busca más la explicación de los acontecimientos mundiales en sí mismos que la respuesta a las causas que los originaron" (2005, p. 33).

Desde la corriente realista, la política exterior es una prerrogativa de los gobiernos centrales y expresa el interés nacional, incluso el enfoque de la política del poder considera las acciones de otras naciones como los principales factores condicionantes de las decisiones en materia de política exterior de un determinado país (Aron, 1985; Morgenthau, 1986). Los primeros estudios que se apartaron de este modelo tradicional de actor racional unificado se dirigieron hacia los modelos burocráticos y organizacionales (Allison, 1988) que tendieron a fragmentar la unicidad de la política exterior diseñada por el Estado central.

Estos avances contribuyeron a reafirmar que "el estudio de lo 'internacional', debe necesariamente reconstruirse según criterios transdiciplinarios. Esta reconstrucción permite dar cuenta de las nuevas configuraciones o contornos flexibles de 'orden mundial', en tanto ubicables a lo largo del eje local-global" (Petrásh, 1998, p. 110). La "reconstrucción de las relaciones internacionales como transdisciplina a lo largo del eje teórico interpretativo local-global (localinternacional-transnacional-global), y su reinserción como área de cruces disciplinarios de las ciencias sociales y de otras áreas del conocimiento científico" (Petrásh, 1998, p. 106). Para esta autora, uno de los retos principales de las relaciones internacionales para convertirse en transdisciplina radica en lo siguiente:

Estimular a las comunidades académicas ubicadas en el campo más amplio de las ciencias sociales para que coadyuven de manera consciente y comprometida a producir conocimiento pertinente al eje teórico interpretativo local-(inter/trans)nacional-global y a la construcción de las relaciones 
internacionales como disciplina de cruce o transdisciplina socio-céntrica. (Petrásh, 1998, p. 125)

Más recientemente ha comenzado a señalarse que "a final de cuentas la importancia reside en situar la agencia del sujeto de investigación, entendiendo su situación dentro de la complejidad de la estructura internacional. Así pues, existen múltiples escalas para explicar el medio internacional" (Lara, 2019, p. 24). "En la medida en que aceptemos que las escalas son constituidas y reconstituidas en la dualidad agencia-estructura, la partición entre los niveles local, regional, nacional e internacional es simplemente una herramienta heurística" (Del Cerro, 2004, p. 206-207). En este sentido, Díaz Abraham concluye:

Hay que reconocer que en términos generales el transnacionalismo señala de forma acertada la importancia de la interdependencia en cuanto elemento dinámico que ha transformado al sistema internacional en un ámbito más abierto en términos de participación de distintos agentes. (2005, p. 58)

De igual forma, el transnacionalismo no pretende sustituir en sus análisis al Estado como actor internacional, pero niega la supremacía o exclusividad concedida por el realismo. El transnacionalismo no acepta la excesiva compartimentación realizada por el análisis sistémico y rechaza las concepciones más radicales de las teorías de la dependencia; proponen, como alternativa, un estudio de la interdependencia. En un anterior trabajo, Luna Pont reafirmaba:

En términos generales, el transnacionalismo aspira a recuperar el estudio de los flujos relacionales que rebasan las fronteras estatales y que se desarrollan entre grupos sociales distintos de los Estados. Estiman que los cambios acaecidos en la sociedad internacional, tales como las mejoras de los medios de comunicación y de transporte, la expansión mundial de la economía de mercado, etcétera, potencian el protagonismo internacional de las personas a través de grupos u organizaciones intermedias que, en gran medida, escapan al control centralizado de los gobiernos, configurando un entramado de relaciones con contornos de alta complejidad. (2011, pp. 70-71)

De acuerdo con García Segura:

[El transnacionalismo permitió] incluir toda una amplia gama de entidades bajo el concepto de actor internacional, son todas aquellas que a lo largo de los años setenta se manifestaron activas sobre la escena internacional: empresas multinacionales, grupos de presión, organizaciones no gubernamentales, ministerios o agencias gubernamentales, etc. A partir de los años ochenta y con un enfoque diferente del transnacionalismo, aparecen una 
serie de trabajos teóricos sobre actores internacionales que han centrado su atención en un fenómeno que se ha extendido notoriamente en la última década: el fenómeno subestatal. (1992, p. 21)

El alemán Karl Kaiser, considerado uno de los padres del transnacionalismo junto con Robert Keohane y Joseph Nye, indicó que nunca el modelo estatocéntrico había existido en estado puro en la historia (salvo en los siglos XVIII y XIX en los que la situación internacional se aproximó a tal esquema) y criticó el olvido que especialistas habían tenido respecto a la multiplicidad de interacciones a través de las fronteras, los grados de interpenetración e interdependencia y la importancia de los actores no estatales (Kaiser, 1969). Desde esta perspectiva, el concepto de política internacional de basamento realista no refleja la complejidad del sistema internacional. El modelo que mejor explica las relaciones y dinámicas de la sociedad internacional es el de la política multinacional, el que comprende los procesos en los cuales las burocracias públicas reparten valores en los marcos de toma de decisiones que están en interpenetración a través de las fronteras nacionales (Kaiser, 1969). A partir de esta interpretación, el autor estructura tres modelos: la toma de decisión multiburocrática, la integración y la política transnacional (Del Arenal, 1990).

Por aquel entonces, los estudios internacionales empezaron a considerar los denominados nuevos actores internacionales, principalmente a partir de la gravitación de las empresas multinacionales, el surgimiento de nuevas organizaciones internacionales, la conformación de organizaciones no gubernamentales y la emergencia de movimientos sociales transnacionales (Mansbach, Fergunson y Lampert, 1976). La complejidad era tal que las concepciones más tradicionales ya no lograban explicar el surgimiento, desarrollo e intensidad de las relaciones internacionales solo desde la óptica de las relaciones interestatales.

La teoría de la interdependencia compleja comienza a cuestionar el modelo del Estado-Nación como actor unitario de la realpolitik; se genera un incremento en el número y tipo de actores internacionales, una agenda internacional sin jerarquías o con una jerarquía más difusa en la política mundial de la que emergían múltiples canales de relacionamiento que podían ser interestatales, transgubernamentales o transnacionales; y en la que tendía -por un lado- a devaluarse, de manera creciente, el uso de la fuerza militar y -por el otro lado-a aumentar la importancia de los factores económicos en el sistema internacional (Keohane y Nye, 1988).

Tras la crítica al actor principal de la realpolitik y la fragmentación de su imagen, emergieron los análisis que se vinculaban con los "múltiples canales" de 
relacionamiento que podían ser interestatales, transgubernamentales o transnacionales (Keohane y Nye, 1988).

[Los] canales múltiples conectan las sociedades; los mismos incluyen tanto nexos informales entre elites gubernamentales como acuerdos formales entre agencias de servicio exterior, nexos informales entre elites no gubernamentales (cara a cara y mediante telecomunicaciones) y organizaciones transnacionales (tales como bancos o corporaciones multinacionales). Estos canales comprenden relaciones interestatales, transgubernamentales y transnacionales. Las relaciones interestatales son los canales normales supuestos por los realistas. Las relaciones transgubernamentales aparecen cuando se flexibiliza el supuesto realista de que los Estados actúan coherentemente como unidades; las relaciones transnacionales surgen cuando se flexibiliza el supuesto de que los Estados son las únicas unidades. (Keohane y Nye, 1988, p. 41)

Si bien estos autores nunca escribieron sobre paradiplomacia, contribuyeron a comprender que existe una parte importante de relaciones que se desarrollan entre los Estados que no son decididas o ejecutadas por los órganos centrales de sus respectivas administraciones sino por "subunidades" del Estado que operan en el exterior, sin una intervención directa del gobierno central. Para Luna Pont estas relaciones pueden implicar la coordinación política, es decir, "actividad orientada a facilitar la implementación o ajustes de políticas en ausencia de directivas precisas provenientes de la esfera nacional", llevada a cabo entre oficiales de distintas burocracias, que una vez "regularizada tiene la capacidad de afectar las expectativas y preferencias de los actores involucrados y de dar lugar a la formación de redes transnacionales de elites" (2011, p. 73):

[Por ello] la construcción de coaliciones transgubernamentales tiene lugar cuando las subunidades construyen coaliciones con agencias de otros gobiernos contra elementos de sus propias estructuras administrativas a los fines de influir en las decisiones gubernamentales. Para que esto pueda suceder los autores identifican una serie de prerrequisitos: los contactos frecuentes, la existencia de intereses comunes entre las subunidades mayor que aquella con agencias de sus propios Estados y un bajo control por parte del Ejecutivo central para permitir tales contactos. (Luna Pont, 2011, p. 73)

En síntesis, Keohane y Nye (1988) nos permiten entender que: "la disponibilidad de socios en las coaliciones políticas no se encuentra necesariamente limitada por las fronteras nacionales, tal como supone el análisis tradicional" (Keohane y Nye, 1988, p. 52). De igual forma es importante considerar lo siguiente: 
Los canales múltiples de contacto de la interdependencia compleja no se encuentran limitados a los actores no gubernamentales. Los contactos entre las burocracias gubernamentales encargadas de tareas similares pueden no sólo alterar sus perspectivas, sino también llevar a coaliciones transgubernamentales sobre cuestiones políticas específicas. Para mejorar sus oportunidades de éxito, los organismos gubernamentales procuran atraer como aliados a actores de otros gobiernos a sus propios procesos de toma de decisiones. (Keohane y Nye, 1988, pp. 52-53)

Junto a la concepción de burocracias públicas de Kaiser (1969) y el reconocimiento de los canales transgubernamentales de Keohane y Nye (1977), se introdujeron las líneas de análisis sobre los actores gubernamentales domésticos a partir de las cuales, en gran parte, se comenzarían los estudios sobre la acción internacional de los gobiernos subnacionales. Si bien Keohane y Nye no abordaron esta última cuestión como tal, contribuyeron con sus estudios a la apertura de una nueva perspectiva en el campo disciplinar de las Relaciones Internacionales en donde se pondría nuevamente de relieve la capacidad del actor por sobre la calificación de entidad soberana (Oddone, 2016).

Se destacaba, así, la importancia de la capacidad o de la habilidad versus la definición o calificación de entidad soberana: "cuando entidades no estatales son capaces de afectar el curso de los acontecimientos internacionales se convierten en actores y, por tanto, entran en competencia con el Estado-nación" (García Segura, 1993, p. 16). Los primeros estudios tendieron a suponer que el accionar internacional subnacional entraba en competencia con el Estado. La relación de competencia o colaboración entre gobierno central y unidades subnacionales comenzaría a ser estudiada tiempo después, particularmente a partir de los estudios de paradiplomacia a mediados de los años ochenta.

Si en los enfoques más tradicionales la categoría de actor se define a partir de un criterio jurídico-formal, que la asocia a la de sujeto de derecho internacional público, en los enfoques posteriores al transnacionalismo, la justificación empieza a incorporar criterios de tipo funcional que marcarán fuertemente los debates acerca de la definición del actor y sus capacidades de agencia para la actividad paradiplomática. Vale decir, la autonomía sucederá a la soberanía como categoría de análisis y se valorará la habilidad de los actores para provocar consecuencias en la política mundial (Alvarez, Luna Pont y Oddone, 2019).

En cuanto a su clasificación como actores internacionales, las unidades subnacionales han sido abordadas como actores internacionales mixtos (Hocking, 1996). Especialmente en los Estados federados, las unidades subnacionales constituyen polities genuinas, en el sentido de que son mucho más que una di- 
visión administrativa del gobierno central y disponen de una serie de atributos (capacidad normativa, territorio, sistema de partidos, identidad colectiva, etc.) cercanos a los de los Estados soberanos. También pueden adoptar estrategias internacionales con mayor libertad que estos, pueden escoger en qué ámbitos quieren estar presentes, colaborar con acciones, combinar instrumentos y crear alianzas propias del mundo gubernamental y del no gubernamental de forma continua o discontinua (Sánchez Cano, 2015).

Siguiendo la conocida distinción de Rosenau (1990) entre actores sovereigntybound y sovereignty-free, se trataría de actores híbridos, parcialmente libres de los rasgos propios de la soberanía y de sus diversas implicaciones. Esta condición le aporta una calidad única a su acción exterior; ya que estos actores híbridos exploran los límites entre las distinciones convencionales -pero a menudo engañosas- entre actores estatales y no estatales y han podido desempeñar una variedad de roles en distintos ámbitos políticos. La capacidad de combinar estrategias, alianzas e instrumentos de uno u otro de los dos mundos de la política mundial -el estatocéntrico del Estado nacional y el policéntrico de los actores no estatales- les provee un carácter único (Rosenau, 1988).

\section{Fragmentación de agenda, poderes de agencia de Estado central y sur- gimiento de los nuevos actores}

No siempre el término utilizado para analizar la complejidad del nuevo escenario internacional fue el de interdependencia. Al mismo tiempo que escribían Keohane y Nye (1977), lo hacía Manning (1977), denominando a la nueva realidad como interméstica (intermestic). Se resumía en un único término a las características de los nuevos temas emergentes en la política internacional que, si bien se trataban a nivel internacional, afectaban directamente los intereses políticos y económicos locales.

De acuerdo con Manning: "La naturaleza de los asuntos internacionales contemporáneos está marcada por ser simultánea, profunda e inseparablemente internacional cuanto doméstica" (Manning, 1977, p. 309). A su vez, este concepto de interméstico se vincula con anteriores estudios como la segunda imagen invertida de Peter Gourevitch. "El sistema internacional no es sólo una consecuencia de la política y estructuras domésticas, sino una causa de las mismas" (1996, pp. 23-24); por ello, "las relaciones internacionales y la política doméstica están tan interrelacionadas que deberían ser analizadas simultáneamente, como un todo" (1996, p.v67). Cohn y Smith plantean:

El alcance de las relaciones internacionales se ha expandido dramáticamente a medida que ha aumentado la interdependencia global, abarcando áreas 
políticas 'nuevas' como la contaminación ambiental, los derechos humanos, la inmigración, la inestabilidad monetaria y comercial y el desarrollo sostenible. A diferencia de los asuntos estratégicos/de seguridad tradicionales, estos nuevos problemas son de naturaleza interméstica; es decir, son 'a la vez profundos e inseparables tanto a nivel nacional como internacional'. (1996, p. 25)

Existe consenso en afirmar que, probablemente, haya sido James Rosenau uno de los primeros y principales autores en valorar la aplicación del concepto interméstico en su relación con las dimensiones subnacionales.

En resumen, una gran cantidad de dinámicas han aumentado enormemente los flujos transfronterizos y las fronteras entre el exterior y el nacional se han vuelto más porosas. Si el colapso del tiempo y la distancia, las organizaciones subnacionales y los gobiernos que una vez operaron dentro de los límites de las fronteras nacionales están ahora tan inextricablemente conectados a partes del mundo lejanas, las jurisdicciones legales y geográficas en las que están ubicadas son cada vez menos importantes. (Rosenau, 2003, p. 227)

De acuerdo con Rosenau, las capacidades analíticas están arraigadas en el territorialismo metodológico, cuestión que lleva a delimitar cada problema en un espacio geográfico o espacial dado, ocasionando dificultades en la comprensión realidades intermésticas. Para este autor, el personal funcionario no es libre de pensar en contextos alternativos porque está "atrapado por la territorialidad de su poder" (2003, pp. 226-227).

Vilma Petrásh (1998) remarca que ante el surgimiento del concepto de interméstico se profundizan las categorías híbridas de análisis y conceptualización. Los análisis híbridos impactaron así sobre la interpretación del desempeño de actores-agentes, estructuras, fenómenos y procesos. Según la autora, esto se evidencia en la interpretación de la "nueva frontera" de James Rosenau y del "espacio social transnacional" de Ulrich Beck. Petrásh también incluye el término de glocalización acuñado por Roland Robertson dentro de este proceso de hibridación. Sin embargo, tal como alertaba Putnam (1988), la política interna y las relaciones internacionales a menudo están enredadas y las teorías propuestas no logran resolver esa complicada maraña.

Chadwick Alger (1977) fue, probablemente, quien primero hizo un llamado para orientar las investigaciones internacionales desde lo local y, luego, a las ciencias sociales para abordar los nexos locales-globales en las relaciones internacionales contemporáneas (Martín López y Oddone, 2010). En el primer caso, centran las ciudades - de perfil industrial principalmente- como unidades de 
aproximación de la política mundial (Alger, 1977; 1988 y 1990). Advertía que las ciudades desempeñaban un papel importante en la política global, debido a que son núcleos creadores de nuevas tecnologías y cultura que se expanden a partir de ellas; son como nodos de los sistemas internacionales que facilitan la interconexión y cuarteles generales desde donde se ejerce la idea del control tanto gubernamental como no gubernamental.

En el caso de los nexos locales-globales, advierte la necesidad de que las ciencias sociales incorporen, en forma sistemática, el análisis del impacto de fenómenos globales en las sociedades:

Impedir el estudio y hasta la percepción de las relaciones entre los asentamientos humanos y los fenómenos mundiales corresponde a una concepción del mundo como un sistema de Estados ... que ha impedido también a los especialistas seguir el curso de las actividades locales que atraviesan las fronteras de los Estados. (Alger, 1988, p. 339)

Luego de identificar las limitadas perspectivas de análisis existentes sobre dicho nexo y las variadas formas en que cuestiones internacionales influyen en los escenarios locales -denominadas por el autor como "formas de intrusión"-, concentra su atención en las respuestas locales a problemas internacionales con impacto local y en la intervención de las autoridades locales sobre problemas relacionados con la política exterior de los Estados (Alger, 1988). En la década de 1990 se profundizaría esta línea de trabajo en el marco de los estudios sobre la globalización y del fenómeno de la glocalización, término acuñado por Roland Roberston.

Según el Oxford Dictionary of New Words, el término glocal y el término glocalización se "forman combinando global y local hasta realizar el término compuesto" (Tulloch, 1991, p. 134). Como sintetiza el propio Robertson (2000):

La necesidad de introducir con firmeza en la teoría social el concepto de glocalización surge de diferentes consideraciones. Gran parte del discurso sobre la globalización ha tendido a asumir que se trata de un proceso superador de lo local, incluyendo aquí a lo local a gran escala ... como se ha manifestado en los diversos nacionalismos .... Tal interpretación descuida dos cosas. Primero, que la extensión de lo que se tiene por local se ha construido en gran parte sobre una base que va más allá de lo local... o que lo supera .... En segundo lugar, mientras que se ha prestado en estos asuntos creciente interés a las consideraciones espaciales y se ha dado una mayor atención a los estrechos vínculos entre las dimensiones espaciales y temporales de la vida humana, esas consideraciones apenas han tenido impacto, ..., en el debate acerca de la globalización ..., se ha hecho poco esfuerzo en conectar la 
discusión en tiempo-espacio con el ... universalismo-particularismo. (2000, pp. 214-215)

En los ochenta, Ivo Duchacek intervendría en el debate, centrando su análisis en los Estados federales, proponiendo analizar las múltiples voces que se escuchan en el ámbito internacional, entre las que prestaba particular atención a los actores gubernamentales subnacionales, los que posteriormente serían considerados entre los "perforadores" de la soberanía estatal. Esto llevó a considerar los tipos de régimen como condicionantes en la elaboración y gestión de políticas exteriores. De la mano de los enfoques del neofederalismo, comienzan a abordarse la dimensión interna -disposiciones constitucionales, el reparto de competencias, las modalidades de relaciones intergubernamentales, etc.- en los distintos modelos de federalismo, como así también los cambios operados en su dimensión externa: atribuciones, capacidades y recursos que disponen los distintos niveles de gobierno para el desarrollo de relaciones internacionales, el marco legal y las formas de articulación entre los distintos niveles gobierno en esta materia ( Forsyth, 1996; Levi,1987, Pinder, 1993).

Los estudios de Ivo Duchacek, Hans Michelmann y Panayotis Soldatos desarrollados a lo largo de las décadas de 1980 y 1990 cuestionan el presupuesto de que el gobierno nacional debe tener una sola voz en la arena internacional y una política exterior unificada que refleje la diversidad interna. Para explicar tal fenómeno de penetración de las fronteras nacionales, Duchacek, Latouche y Stevenson (1988) las entienden como tamices (sieves), frente a la imagen realista de las fronteras como caparazones (hard shells) impenetrables. La interdependencia acaba con los compartimentos estáticos, los que empiezan a ser interferidos y penetrados (marbled) por otros actores que incluyen a los gobiernos subnacionales. Los estudios clasifican los diferentes perforadores; incluso identifica como categoría de análisis a los perforadores subnacionales, entre los que se destacan los gobiernos locales, municipales o regionales, y las comunidades territoriales o etnoterritoriales. Los otros perforadores son la oposición; los grupos de intereses privados y los inmigrantes.

De acuerdo con Michel Acuto (2013) y Ray Freddy Lara (2019), el concepto de paradiplomacia, desde la interpretación de Duchacek, fortalece los poderes de agencia de las unidades subestatales en el sistema internacional.

Ivo Duchacek (1986) revivió la idea de paradiplomacia como una forma de agencia política de las entidades subnacionales. Donde estas perforan la soberanía (Duchacek, Latouche y Stevenson, 1988) de los Estados, priorizando sus intereses particulares a través de conexiones transfronterizas, transregionales y globales. (Acuto, 2013, p. 8) 
La fragmentación de la agenda global impacta, por tanto, en los poderes de agencia de los diferentes actores, incluyendo especialmente los gobiernos subnacionales. Y, desde esta perspectiva, sus comportamientos internacionales comienzan a ser abordados como expresión de paradiplomacia.

Ya no se trata de comprender el fenómeno meramente como expresión de una "acción" (establecimiento de contactos, relaciones entre burocracias, reacción a la influencia de factores externos o frente a la complejidad del escenario internacional), sino de un tipo de "política pública" definida como "las entradas directas e indirectas de los gobiernos no centrales al campo de las relaciones internacionales y de su vinculación con la política exterior" (Duchacek, 1990, p. 15). El autor ponía énfasis en la palabra "para" ya que entendía que esta capacidad de los gobiernos locales era "paralela", es decir, "subsidiaria o accesoria" (Duchacek, 1990, p. 25) respecto a los gobiernos centrales. Duchacek (1988) distingue diferentes tipos de paradiplomacia:

La regional, a través de las fronteras entre gobiernos no-centrales vecinos; la transregional, que refiere a contactos usualmente institucionalizados entre gobiernos no centrales que no son vecinos geográficamente, pero cuyos gobiernos centrales si lo son; y la global, que involucra contactos directos entre gobiernos no centrales de una nación con contrapartes subnacionales en otras naciones distantes. (1988, pp. 12-13)

Diferentes estrategias que podrían enmarcarse en el concepto de paradiplomacia han sido llevadas a cabo con el objetivo de aumentar los márgenes de acción y autonomía de los gobiernos subnacionales; y han generado, de esta manera, una serie de modificaciones en las concepciones diplomáticas más tradicionales, que legitiman su propio accionar y los intereses manifiestos en dinámicas multinivel y multiactor.

No debe pensarse el ascenso de la paradiplomacia como el declive de la diplomacia estatal, sino que se trata de un conjunto de prácticas de interrelación transnacionales que se superponen parcialmente a aquella y complejizan el escenario político mundial. Asimismo, estas prácticas no constituyen una (re) producción mimética de los procesos tradicionales de la política exterior, sino que los estilos y estrategias de actuación están marcados por objetivos y motivaciones mucho más concretas y delimitadas. (Ferrero, 2006, p. 7)

Dentro de las dimensiones propuestas para el análisis de la paradiplomacia desde la perspectiva del actor internacional, vale la pena también relevar la aproximación vinculada con la dimensión organizacional-administrativa que es aquella que se enfoca en la unidad geográfico-política que permite canalizar un proyecto 
político local que incluye la internacionalización. Esta dimensión incluye el análisis de la organización interna del gobierno local o regional en relación con la política internacional subnacional a partir del estudio de los organigramas institucionales, las funciones de las oficinas de relaciones internacionales, los recursos humanos que las conforman, los recursos presupuestarios y la infraestructura de servicios que la caracteriza (Vigevani, 2006; Calvento, 2015).

La fragmentación del Estado central se convirtió, así, en un tema abordado desde múltiples corrientes dentro de la disciplina de las relaciones internacionales, dada la dispersión geográfica de las actividades económicas, característica de la globalización; y de forma derivada, las unidades subnacionales se transformaron en objeto de estudio como actores relevantes de la política mundial. Los procesos de descentralización y la delegación de parte de la soberanía hacia instituciones supranacionales profundizaron los debates acerca del papel del Estado central como único actor de las relaciones internacionales, y su automática vinculación con un territorio.

Es en este contexto, las diferentes aproximaciones fueron confrontándose con teorías que abordaban la territorialidad desde una perspectiva moderna vinculada a la economía no territorial. Por ello, algunos autores propugnaron por el abandono del término de relaciones internacionales sustituyéndolo por el de políticas post-soberanas (MacCormick, 1993) o simplemente el de política global (Ferguson y Mansbach, 1996), en la que todos los Estados deben compartir autoridad e influencia con "otras entidades políticas" (Ferguson y Mansbach, 2002, p. 103), y a partir de ello, entender el rompecabezas global (Mansbach, 1997).

Tal como sostiene Sánchez Cano (2015), las ciudades y las regiones quedan en el centro de la escena, estudiadas como lugar privilegiado de la territorialización de las dinámicas globales y como actores en términos de constituir el entorno territorial, humano y productivo en el que operan los procesos globales; en lo económico como espacios de acumulación que aspiran a transformarse en un nodo insertado en la nueva economía global -con un alto componente tecnológico e informacional, y en un mundo crecientemente urbanizado- y en el mercado global, a partir de las pujas por atraer los factores de competitividad: inversiones, negocios, sedes corporativas, etc. (Sánchez Cano, 2015). La nueva competitividad entre ciudades mundiales, internacionales o globales se proyecta al escenario internacional, y comienza a ocupar un lugar central en la forma de concebir y abordar la gestión internacional subnacional (Sassen, 1994).

[De todas maneras, el territorio nunca perdió] una función central en el vínculo sociedad-mercado global, explicando los cambios generados por el Estado, los procesos de descentralización, y las capacidades de respues- 
ta desde lo subestatal y sus posibles articulaciones; tanto a nivel nacional como transnacional, interregional e internacional, para dar una repuesta a la complejidad de esos escenarios. (Oddone y Álvarez, 2019, p. 54)

Las entidades subnacionales se han visto así "obligadas a improvisar y desarrollar formas hibridas de diplomacia, la fusión de prácticas diplomáticas tradicionales y alternativas ha permitido el establecimiento de relaciones exteriores, siendo el aspecto territorial, su distintivo frente a otros actores no estatales" (Duran, 2012, p. 5). De igual modo, la proliferación en la bibliografía especializada del análisis de redes de gobiernos locales y regionales generó importantes análisis de casos sobre redes territoriales como estrategia clave en la articulación de actores subnacionales entre ellos y de vinculación global con el sistema internacional. Los enfoques de la gobernanza global y la gobernanza multinivel dan cuenta de ello, e introducen las redes de ciudades como actores clave en la "hechura" de la política mundial.

Desde el campo del reflexivismo se introducen nuevos matices al análisis de la subjetividad internacional de estos actores subestatales. Desde el constructivismo, por ejemplo, se abordan los procesos comunicativos entre los actores estatales y los no estatales a partir de los cuales se producen las interpretaciones dominantes de las realidades, las orientaciones de políticas e ideas acerca de cómo gobernar la política mundial. Desde el supuesto de que ideas, identidades e intereses son socialmente construidos y endógenos al proceso de interacción, se analizan los procesos comunicativos que crean y transforman la relación entre los actores y, en particular, las pujas por las interpretaciones dominantes (Lara Pacheco y Cerqueira Torres, 2017; Simmerl, 2011). Desde esta perspectiva, la subjetividad sociológica y jurídica de los actores subestatales es la resultante de un proceso de formación y lucha discursiva que los actores deben rearticular para participar exitosamente (Lara Pacheco y Cerqueira Torres, 2017).

Como se ha señalado, algunos analistas encuentran en la paradiplomacia el fortalecimiento de los poderes de agencia de las unidades subestatales en el sistema internacional (Acuto, 2013; Duchacek, 1986; Lara, 2019). Esta cuestión introduce al análisis una dimensión de tales desempeños que no ocupó, hasta años recientes, un lugar importante en la reflexión: la capacidad de agencia o, en otros términos, su actorness o ponderación de la entidad como actor a partir de la gravitación en el plano internacional.

Esta aproximación remite a una discusión de base ontológica sobre la ponderación de los agentes y las estructuras en la teoría de las relaciones internacionales. Por un lado, quienes sostienen que las explicaciones científicas deberían ser reducibles a las propiedades e interacciones de los individuos, otorgando un 
status ontológico secundario a las estructuras al afirmar que estas solo tienen efecto sobre el comportamiento y constriñen las opciones de los actores; y por otro lado, quienes entienden que los efectos de las estructuras contribuyen a la construcción de los agentes, tienen efectos sobre sus propiedades, es decir, sobre las identidades e intereses de los propios agentes. Este tipo de abordaje, entre otras cosas, da lugar a la ponderación de los actores en términos de su cualidad, entidad y capacidad/habilidad de impacto en la política mundial a partir de su capacidad de agencia.

El concepto de actorness se entiende como la "capacidad de comportarse de manera activa y deliberada -esfuerzo conciente y voluntario- en relación con otros actores en el ámbito internacional" y de producir impacto ${ }^{4}$. La capacidad de agencia puede ser entendida como el resultado de un proceso de dinámico entre por lo menos tres elementos: actorness, presencia y purposive actorness (actorness intencional); en donde presencia expresa un sentido de autoafirmación y la forma en que se es percibido, reconocido y legitimado por otros actores; y purposive actorness, la intención consciente de producir impactos en el orden mundial desde los propios valores e intereses (Groen y Niemann, 2011).

Por lo demás, la perspectiva de actorness se complementa con aquellas que trabajan la efectividad, esto es, la consideración del logro de resultados (Wunderlich, 2008). Ello supone abordar la paradiplomacia no simplemente como "reactiva" frente a presiones o intrusiones del entorno internacional, sino también como expresión de empoderamiento de estos actores a partir de su voluntad de realizar reivindicaciones y de influir en la gobernanza global promoviendo cambios en las reglas de juego o en la agenda global desde la mirada local. La evaluación de los requerimientos para dotar de efectividad la acción queda en el centro del estudio de las características propias (autopercibidas) y percibidas por pares y demás actores del sistema internacional.

\section{Conclusiones}

Desde la mirada del mainstream, la heterogeneidad de los casos de paradiplomacia dificulta todavía la elaboración de una teoría global sobre la acción internacional de los gobiernos locales (Gely, 2016). Sin embargo, es dable destacar que "los cambios en las asunciones e interpretaciones epistemológicas que ayudan a formular y estructurar el entendimiento y la acción colectiva constituyen la noción más significativa de aprendizaje en relaciones internacionales" (Adler y Haas, 2009, p. 164). Los procesos de aprendizaje institucionales, especial-

4 Sjöstedt (1977) definía esta categoría con relación a actores como la Comunidad Europea. 
mente en materia de paradiplomacia, pueden caracterizarse desde la perspectiva del isomorfismo, en el sentido de que las instituciones tienden a moldearse siguiendo instituciones similares, se desempeñan en su mismo campo de acción o son percibidas como legítimas o exitosas (Di Maggio y Powell, 1983).

La inexistencia de una única vía para el desarrollo de la paradiplomacia implica que su diseño dependerá de la capacidad de concertación de las autoridades locales, la movilización de otros actores socios, los recursos disponibles, sus trayectorias de gobierno y la acumulación de otras experiencias, así como la posibilidad de imitar estructuras parecidas. La exultante conformación de redes de ciudades a lo largo de los últimos años y a partir de ejes temáticos específicos es fiel prueba de ello.

El creciente interés por el estudio de la paradiplomacia y por construir argumentos teóricos de peso que contribuyan a entender que los gobiernos locales y regionales son actores importantes del sistema internacional ha encontrado, a lo largo de los últimos años, múltiples apoyos y exponentes a escala global. Sin duda, la construcción holística de la Agenda 2030 y de los Objetivos de Desarrollo Sostenible (ODS), con la participación de gobiernos municipales e intermedios, ayudó a consolidar los aportes surgidos desde una perspectiva bottom up en la gobernanza global.

De acuerdo con los diferentes análisis realizados por varias redes globales de ciudades, se estima que de las 169 metas fijadas en los ODS los gobiernos locales tienen un rol que cumplir en -al menos- 93 de ellas. Este espacio global ofrece la oportunidad de fortalecer las capacidades internas, así como los recursos técnicos y financieros para consolidar los mecanismos de intercambio de buenas prácticas; y, de esta manera, visibilizar los aportes sustantivos que permitan avanzar en el proceso interméstico descripto como un legítimo reclamo para obtener una "silla local en la mesa de las decisiones globales".

En este proceso de mayor internacionalización y de aporte subnacional/subestatal a la gobernaza global, sin lugar a duda, quienes plantean teorías y estudian la disciplina de las relaciones internacionales tienen mucho que decir. De igual manera, es oportuno que aquellos investigadores e investigadoras que sostienen el accionar paradiplomático se constituyan como una sólida y legítima comunidad epistémica (Haas, 1992), para ejercer una influencia en la renovación de las políticas públicas, y permitan fortalecer y encausar los aportes de la paradiplomacia a la gobernanza global.

La implementación de las nuevas capacidades de agencia de los gobiernos subnacionales, la difusión del análisis de experiencias del tipo local-central/global- 
local, la coordinación de políticas transnacionales y, en general, toda la difusión de estudios comparados y prospectivos desarrollados por comunidades y redes de personas expertas, juegan un papel fundamental en demostrar no solo las características del ejercicio legítimo de presión intelectual (lobby) al interior de la disciplina, sino también sobre los entes decisores políticos para la difusión de las "innovaciones intelectuales [que] ayudan a los gobiernos a redefinir sus expectativas, a alcanzar comprensiones comunes y a coordinar sus acciones de acuerdo con estas categorías" (Adler y Haas, 2009, p. 157).

En este sentido, la comunidad epistémica de internacionalistas, que estudia y se siente comprometida con estos temas, necesita consolidar su agenda transnacional y persistir en los vínculos políticos con esta agenda a partir de la difusión y socialización de sus estudios, su asesoramiento y asistencia técnica en los procesos de negociación y toma de decisiones de los gobiernos locales y regionales. Además, vale la pena mencionar que la paradiplomacia se ha convertido en un campo de acción profesional para gran cantidad de recientes personas graduadas de la carrera de Relaciones Internacionales y Ciencias Políticas.

Hoy, más que nunca, la diversidad teórica de las relaciones internacionales es una de sus fortalezas como campo disciplinar, y los avances realizados hacia un multiparadigmatismo ponen de relieve que las relaciones internacionales constituyen una globalidad que jamás podrá ser analizada como tal; por lo que siempre es oportuno recordar que más allá de "nuestra sofisticación metodológica, probablemente no comprendemos la política internacional contemporánea como debiéramos; debido a que el desarrollo teórico de nuestra disciplina va actualmente por detrás de la cambiante realidad de la práctica diaria de los asuntos internacionales" (Puchala y Fagan, 1974, p. 247). De igual manera sucede en muchas disciplinas.

\section{Referencias}

Aaron, R. (1985). Paz y guerra entre las naciones. Madrid: Alianza Editorial.

Acharya, A. y Buzan, B. (Eds.). (2010). Non-Western International Relations Theory. Perspectives on and beyond Asia. London: Routledge.

Acharya, A. y Buzan, B. (2019). The Making of Global International Relations: Origins and Evolution of IR at its Centenary. Cambridge: Cambridge University Press.

Acuto, M. (2013). Global Cities, Governance and Diplomacy. The Urban Link. London-New York: Routledge. 
Adler, E. y Haas, P. (2009). Conclusión: Las comunidades epistémicas, el orden mundial y la creación de un programa de investigación reflectivo. Relaciones Internacionales, (12), 145-169.

Alger, C. (1977). The impact of Cities on International System. Eukistics, 44 (264), 243-253.

Alger, C. (1988). Las nexos locales-mundiales: Su percepción, análisis y enfoques. Revista Internacional de Ciencias Sociales, (117), 339-360.

Alger, C. (1990). The World Relations of Cities: Closing the Gap between Social Science Paradigms and Everyday Experience. International Studies Quarterly, 34 (3), 493-518.

Allison, G. (1988). La esencia de la decisión. Análisis de la crisis de los misiles en Cuba. Buenos Aires: Grupo Editor Latinoamericano.

Batta Fonseca, V. (2013). Impulso a la enseñanza de la prospectiva en Relaciones Internacionales. Revista de Relaciones Internacionales de la UNAM, (116), 43-73.

Bueno, I. (2012). Paradiplomacia econômica: Trajetórias e Tendências da Atuação Internacional dos Governos Estaduais do Brasil e dos Estados Unidos. Brasília: Verdana.

Caballero, S. (2009). Comunidades epistémicas en el proceso de integración regional sudamericana. Cuadernos sobre Relaciones Internacionales, Regionalismo y Desarrollo, 4 (8), 11-26.

Calvento, M. (2015). Hacia un concepto multidimensional de la política y la gestión internacional subestatal. En Calvento, M. (Comp.), Procesos y actores en la gestión de la política internacional subestatal (pp.15-40). Tandil: Universidad Nacional del Centro de la Provincia de Buenos Aires.

Cohn, T. y Smith, P. (1996). Subnational Governments as International Actors: Constituent Diplomacy in British Columbia and the Pacific Northwest. BC Studies. The Britich Columbia Quaterly, (110), 25-59.

Couperus, S. (2011). Between Vague Theory and Sound Practical Lines: Transnational Municipalism in Interwar Europa. En Laqua, D (Ed.), Internationalism reconfigured. Transnational ideas and movements between the world wars (pp. 67-89). London: IB Tauris.

Del Arenal, C. (1981). La génesis de la relaciones internacionales como disciplina científica. Revista de Estudios Internacionales, 2 (4), 849-892.

Del Cerro, G. (2004). Ciudades y globalización: Un enfoque teórico. Revista Española de Sociología, (4), 199-218. 
Díaz Abraham, L. (2005). La cooperación oficial descentralizada para el desarrollo: Perfil de su desempeño en los años noventa. Madrid: Universidad Complutense de Madrid.

Di Maggio, P. y Powell, W. (1983). The Iron Cage Revisited: Institutional Isomorphism and Collective Rationality in Organizational Fields. American Sociological Review, 48 (2), 147-160.

Duchacek, I. (1970). Comparative Federalism: the territorial dimension of politics. Nueva York: Holt, Rinehart and Winston.

Duchacek I. (1986). The Territorial Dimension of Politics: Within, Among and Across Nations. London: Westview Press.

Duchacek, I., Latouche, D. y Stevenson, G. (1988). Perforated Sovereignties and International Relations: Trans-Sovereign Contacts of Subnational Governments. New York: Greenwood Press.

Duran, M. (2012). Bridging the gap between Self and Other? Mediterranean Paradiplomacy as Homo-diplomacy. ECPR Antwerp Joint Research Sessions.

Ferrero, M. (2006). La glocalización en acción: Regionalismo y paradiplomacia en Argentina y el Cono Sur Latinoamericano. Revista Electrónica de Estudios Internacionales, (11), 1-22.

Fried, A. (1909). La science de l'internationalisme. En Office Central des Institutions Internationales. Annuaire de la Vie Internationale, 1908-1909, (pp. 2328). Brussels: Office Central des Institutions Internationales..

Forsyth, M. (1996). The political theory of federalism: the relevance of classical approaches. En Hesse, J. y Wright, V. (Eds.), Federalizating Europe? Costs, benefits, and preconditions offederal political systems. Oxford: Oxford University Press

García Segura, C. (1993). La evolución del concepto de actor internacional en la teoría de las relaciones internacionales. Papers: Revista de Sociología, (41), 13-31.

García Segura, C. (1992). La presència de les entitats polítiques subestatals a les Relacions Internacionals. Barcelona: Universitat Autònoma de Barcelona.

Gely, M. (2016). Hacia una mejor conceptualización teórica de la proyección internacional de los gobiernos locales. En González, S., Cornago, N. y Ovando, C. (Eds.), Relaciones transfronterizas y paradiplomacia en América Latina. Aspectos teóricos y estudios de casos (pp. 161-176). Santiago: RIL Editores y Universidad Arturo Prat. 
Gourevitch, P. (1996). La segunda imagen invertida: Los orígenes internacionales de las políticas domésticas. En Gil, O. y Sanz, L. (Comp.), Las fuentes internacionales de las políticas domésticas. Madrid: Zona Abierta.

Groen, L. y Niemann, A. (2011). EU actorness and effectiveness under political pressure at the Copenhagen climate change negotiations. Twelfth European Union Studies Association Conference Boston. Massachusetts.

Haas, P. (1992). Introduction: Epistemic Communities and International Policy Coordination. International Organization, 46 (1), 1-35.

Halliday, F. (2002). Las relaciones internacionales en un mundo en transformación. Madrid: La Catarata.

Hocking, B. (1996). Bridging boundaries: creating linkages. Non-central governments and multilayered policy environments. WeltTrends, (11), 36-51.

Holsti, K. (2005). Obstáculos para el entendimiento en las relaciones internacionales. En Adams, J. (Comp.), Política mundial: Cambio y conflicto. Ensayos escogidos de Kal Holsti. Ciudad de México: Centro de Investigación y Docencias Económicas.

Kaiser, K. (1969). Transnationale Politik: Zu einer Theorie multinationaler Politik. En Czempiel, Ernest Otto (Hrsg.), Die anachronistische Souveränität. Zum Verhältnis von Innen- und Außenpolitik. Köln: Westdeutscher Verlag.

Kehoane, R. y Nye, J. (1988). Poder e interdependencia. La politica mundial en transición. Buenos Aires: Grupo Editor Latinoamericano.

Keohane, R. y Nye, J. (1974). Transgovernmental Relations and International Organizations. World Politics, 27 (1), 39-62.

Knutsen, T. (2008). A lost generation? IR scholarship before World War I. International Politics, 45 (6), 650-674.

Krippendorff, E. (1993). Las relaciones internacionales como ciencia. Una introducción. México: Fondo de Cultura Económica.

Lara Pacheco, R. (2019). La inserción de las ciudades en el medio internacional. Una revisión histórica, teórica y empírica desde las relaciones internacionales. Guadalajara: Universidad de Guadalajara.

Lara Pacheco, R. y Cerqueira Torres, O. (2017). Las ciudades en la gobernanza global multinivel: Una aproximación teórica. Si Somos Americanos, 17 (2), 43-75.

Levi, L. (1987). Recent developments in federalist theory. Il Federalista, 29 (2), 97-136. 
Luna Pont, M. (2010). Perspectivas teórico-conceptuales de las ciudades y los poderes locales dentro de la dimensión subnacional en las relaciones internacionales. En Martín López, M. y Oddone, N. (Comps.), Las ciudades y los poderes locales en las relaciones internacionales contemporáneas (pp. 4982). Granada: Unión Iberoamericana de Municipalistas.

Luna Pont, M. (2011). Las relaciones internacionales subnacionales desde la teoría de las relaciones internacionales. TIP - Trabajos de Investigación en Paradiplomacia, 1 (1), 64-83.

Luna Pont, M. (2019). De IULA A CGLU: Municipalismo internacional, narrativas y momentos. En Alvarez, M., Luna Pont, M. y Oddone, N. (Eds.), AMÉRICA LATINA GLOBAL. Estudios regionales sobre paradiplomacia. Buenos Aires, Editorial de la Universidad Nacional Tres de Febrero (en imprenta).

Mansbach, R., Fergunson, Y. y Lampert, D. (1976). The web of world politics. Nonstate actors in the global system. Englewood Clifs: Prentice Hall.

Martín López, M. y Oddone, N. (Comps.). (2010). Las ciudades y los poderes locales en las relaciones internacionales contemporáneas. Granada: Unión Iberoamericana de Municipalistas.

Marx, V. (2010). Las ciudades y su inserción política en las relaciones internacionales. En Martín López, M. y Oddone, N. (Comps.), Las ciudades y los poderes locales en las relaciones internacionales contemporáneas (pp. 25-48). Granada: Unión Iberoamericana de Municipalistas.

Manning, B. (1977). The Congress, the Executive and Intermestic Affairs: Three Proposals. Foreign Affairs, 55 (2), 306-324.

Morgenthau, H. (1986). Política entre las naciones. La lucha por el poder y la paz. Buenos Aires: Grupo Editor Latinoamericano.

Oddone, N. (2008). La red de mercociudades: Globalización, integración regional y desarrollo local. Valencia: Universidad Politécnica de Valencia.

Oddone, N. (2016). La paradiplomacia desde cinco perspectivas: Reflexiones teóricas para la construcción de una comunidad epistémica en América Latina. Revista de Relaciones Internacionales, 89 (2) 47-81.

Oddone, N. (2019). Estudios sobre paradiplomacia: Su encuadre teórico desde cinco perspectivas. En Bermeo, F. (Coord.), Paradiplomacia y desarrollo territorial (pp. 67-104). Quito, Facultad Latinoamericana de Ciencias Sociales.

Oddone, N. y Alvarez, M. (2019). El lugar del territorio en los estudios paradiplomáticos. En Villarruel, D., et. al. (Coord.), Actores e intereses para la internacionalización del Estado de Jalisco. Guadalajara: Universidad de Guadalajara. 
Petrásh, V. (1998). De lo internacional a lo global. Reconstruyendo los estudios internacionales en los tiempos de post-internacionalización: Aportes para el tercer debate. Disponible en http://www.iaeal.usb.ve/documentos/nro_91/ petrashv.pdf

Pinder, J. (1993). The New European Federalism: The Idea and the Achievements. In Michael B. and Alain-G. G. (Eds.), Comparative Federalism and Federation: Competing Traditions and Future Directions (pp. 45-66). Hemel Hempstead: Harvester Wheatsheaf.

Puchala, D. y Fagan, S. (1974). International Politics in the 1970: The Search for a Perspective. International Organization, 28 (2), 247-266.

Putnam, R. (1988). Diplomacy and Domestic Politics: The Logic of Two-Level Games. International Organization, 42 (3), 427-460.

Premier Congrés International et Exposition Compareé des Villes (1914). Construction des villes et Organisation de la vie communale. Bruselas.

Renouvin, P. y Duroselle, J. (2000). Introducción a la historia de las relaciones internacionales. México: Fondo de Cultura Económica.

Robertson, R. (2000). Glocalización: Tiempo-espacio y homogeneidad-heterogeneidad. Revista Zona Abierta, (92-93), 213-242.

Romero, M. (2004). Una aproximación contextual y conceptual a la cooperación descentralizada. En Romero, M. y Godínez, V. (Eds.), Tejiendo lazos entre territorios. La cooperación descentralizada local Unión Europea-América Latina (pp. 19-54). Valparaíso: Municipalidad de Valparaíso y Diputación de Barcelona.

Rubiolo, F. (2019). Cien años de relaciones internacionales. La Voz, 8 de mayo, disponible en https://www.lavoz.com.ar/opinion/cien-anos-de-relacionesinternacionales

Rosenau, J. (2003). Governance in a new global order. En Held, D.; McGrew, A. (Eds.), The global transformations reader: an introduction to the globalization debat (pp. 223-234). Cambridge: Polity Press.

Rosenau, J. (1988). Patterned chaos in global life: structure and process in the two worlds of World Politics. International Political Science Review, 9 (4), 327364.

Russell, R. (2010). El Estado nación y los actores gubernamentales no centrales: Una relación complementaria. En Maira, L. (Ed.), La política internacional subnacional en América Latin (pp. 83-106). Buenos Aires: Libros del Zorzal. 
Sánchez Cano, J. (2015). Los gobiernos no centrales y sus redes. Análisis de su rol como actores en la gobernanza mundial (Tesis doctoral). Barcelona: Universitat Autònoma de Barcelona.

Sassen, S. (1994). Cities in a world economy. Thousand Oaks: Pine Forge Press.

Simmerl, G. (2011). A Critical Constructivist Perspective on Global Multi-Level Governance. Discursive Struggles among Multiple Actors in a Globalized Political Space (Manuscrito inédito). Berlín: Freie Universität Berlin.

Sjöstedt, G. (1977). The External Role of the European Community. Saxon House, Swedish Institute of International Affairs.

Sodupe, K. (2003). La teoría de las relaciones internacionales a comienzos del siglo XXI. Zarautz: Servicio Editorial de la Universidad del País Vasco.

Speeckaert, G. (1970). Ojeada sobre sesenta años de actividades de la Unión de Asociaciones Internacionales (Documento n. ${ }^{\circ}$ 17). Bruselas: Unión de Asociaciones Internacionales.

Tulloch, S. (Comp.). (1991). The Oxford Dictionary of New Words. Oxford: Oxford University Press.

Tussie, D. (2019). Cien años de relaciones internacionales; ¿qué festejamos? En VI Jornadas Académicas de Licenciatura en Relaciones Internacionales. "Los 100 años de los estudios en Relaciones Internacionales”. Montevideo: Universidad de la República.

Young, O. (1971). The Actors in World Politics. En Rosenau, J; Davis, V. y East, M. (Eds.), The Analysis of International Politics (pp. 125-144). Glencoe: Free Press.

Vigevani, T (Coord.). (2006). Gestão Pública e Inserção Internacional das Cidades. São Paulo: CEDEC, PUC-SP, FGV-SP, UNESP.

Weaver, O. (1996). The rise and fall of the inter-paradigm debate. En Smith, S.; Booth, K. y Zalewski, M. (Eds.), International Theory. Positivism and Beyond (pp. 149-185). Cambridge: Cambridge University Press.

Wunderlich, U. (2008). The EU: A Post-Westphalian Actor in a NeoWestphalian World? En UACES Annual Research Conference. Edinburg: University of Edinburgh. 
